\title{
Generation of $x$-ray radiation in a storage ring by a superconductive cold-bore in-vacuum undulator
}

\author{
S. Casalbuoni, M. Hagelstein, B. Kostka, and R. Rossmanith \\ Institute for Synchrotron Radiation, Research Center Karlsruhe, P.O. Box 3640, D-76021 Karlsruhe, Germany
}

M. Weisser and E. Steffens

Physics Institute II, Friedrich Alexander University Erlangen-Nürnberg, Germany

\author{
A. Bernhard, D. Wollmann, and T. Baumbach \\ Laboratory for Application of Synchrotron Radiation, University of Karlsruhe, Germany
}

(Received 1 May 2005; published 13 January 2006)

\begin{abstract}
The first beam measurements with a cold-bore superconducting in-vacuum undulator in a storage ring are reported. Undulators are $\mathrm{x}$-ray generators in light sources. The physical limitations of these devices limit the intensity and the brilliance of the x-ray beam. At present the undulators are made from permanent magnets. It was shown in earlier papers that at low electron beam intensities superconductive wires in the vacuum beam pipe can overcome the limitations inherent to permanent magnet undulators. It was argued that the use of these novel devices in light sources with high beam currents may be limited by the extreme anomalous skin effect regime in $\mathrm{Cu}$ at $4.2 \mathrm{~K}$, which has so far undergone very little investigation, and the power deposited by the infrared part of the synchrotron radiation. The purpose of this paper is to present measurements of these effects at the synchrotron light source ANKA with stored currents up to $200 \mathrm{~mA}$.
\end{abstract}

DOI: 10.1103/PhysRevSTAB.9.010702

PACS numbers: 07.85.Qe

Brilliance (photons per sec, per $\mathrm{mm}^{2}$, per $\mathrm{mrad}^{2}$, and per $0.1 \%$ bandwidth) and the energy of the photons are the figures of merit of an x-ray beam line in a synchrotron light source. Both parameters are influenced by the electron beam quality and the photon generating device. In modern storage rings and FELs the preferred photon sources are undulators, i.e., magnets with alternating field gradients [1]. Up to now attempts to increase brilliance, and thus the field of application, always focused on improving electron beam quality thereby leading to larger accelerators. However, very little effort was invested in developing new undulator concepts. The undulators were built from permanent magnets. Several years ago it was shown that superconductive undulators can produce higher magnetic fields; however, doubts were voiced whether the superconductive in-vacuum undulators could without a quench survive the heating by the high image currents caused by the anomalous skin effect at $4.2 \mathrm{~K}$ [2] and the wide angle synchrotron radiation produced by the undulator itself. Since only theoretical investigations exist, which are based on various assumptions, it was decided to perform a first experiment. This experiment and the results are described in this paper.

The maximum obtainable field strength in the undulator (for a given period length and a given gap) is a figure of merit of the undulator. This is explained briefly in the following.

The period length of the undulator, the field of the undulator, and the beam energy define the wavelength of the emitted radiation

$$
\lambda=\frac{\lambda_{u}}{2 n \gamma^{2}}\left(1+\frac{k^{2}}{2}+\gamma^{2} \Theta^{2}\right)
$$

$\lambda$ is the wavelength of the emitted radiation, $\lambda_{u}$ the period length of the undulator, $\gamma$ the relative beam energy, $n$ is the harmonic number of the emitted radiation $(n=1,3,5, \ldots$ at the undulator axis), $\Theta$ is the angle between beam axis and photon direction, $k / \gamma$ is the maximum deflection angle in the undulator $k=0.934 . B[T] . \lambda_{u}[\mathrm{~cm}]$ and $B$ is the maximum magnetic field on the beam axis. Conventional undulators are built from permanent magnets. The maximum field strength is obtained when the undulator is in-vacuum: the absence of a vacuum chamber allows the undulator to operate with a smaller gap width $g$. The field strength $B$, as a function of $\lambda_{u}$, depends on the undulator material and the gap of the undulator. For permanent undulators the maximum field $B_{\max }$ is mainly defined by the material properties of the rare earth magnets and, to a certain extent, by the specific design details [3].

$$
B_{\max }=1.55 \exp \left(-\pi g / \lambda_{u}\right),
$$

$k$ in (1) is $\leq 1$ for an undulator. Cooling the permanent magnets to liquid nitrogen temperatures increases the field strength by about $30 \%$ [4].

Should it prove possible to build an undulator with a significantly higher magnetic field than the one described in (2), the same photon energy will be obtained with lower beam energy [see (1)]. The higher the beam energy, the higher the construction costs for a light source.

Around 1990 both Brookhaven [5] and Karlsruhe [6] presented different proposals to replace the permanent 
magnets by superconductive wires or striplines in order to increase the field strength of the undulators. It was later proved experimentally that the field strength of such an undulator exceeds the field strength of permanent magnet undulators by a factor of about two [7] (period length of $14 \mathrm{~mm}$ at $4.2 \mathrm{~K}$ ). By operating this undulator at lower temperatures (1.8 or $2.2 \mathrm{~K}$ ) or using NbSn wires instead of $\mathrm{NbTi}$ wires, there is a potential to produce even higher fields [8]. It also became clear that these high fields can only be obtained when the undulator coils are in-vacuum and positioned as close as possible to the beam. The investigations were performed in two steps.

First, a superconductive in-vacuum undulator was installed in the Mainz microtron MAMI several years ago and successfully tested with beam [9] (50 $\mu \mathrm{A}$ linac type cw beam, beam energy $855 \mathrm{MeV}$ ). In a second step, the central focus of this paper, a storage ring test was prepared at ANKA [10] in the course of which the undulator was tested at currents up to $200 \mathrm{~mA}$. The undulator was built by ACCEL Instruments GmbH, Germany.

Figure 1 shows the schematic layout of the device used for this experiment. The period length is $14 \mathrm{~mm}$ and the undulator has 100 periods. An example of the field measured is shown in Fig. 2 [11].

Figure 2(a) shows the field measurements in the central part of the undulator. Looking at the field minima it is obvious that there are periodic small field variations. A detailed analysis shows that the period of the variations is about 12.5 periods. This is due to the fact that the wires are pressed by a tool into the grooves which has a length of 12.5 periods. The flatness of this tool was about $50 \mu \mathrm{m}$ so that the transverse position of the wires has a similar tolerance and leads to a period phase error of about $2^{\circ}$ in

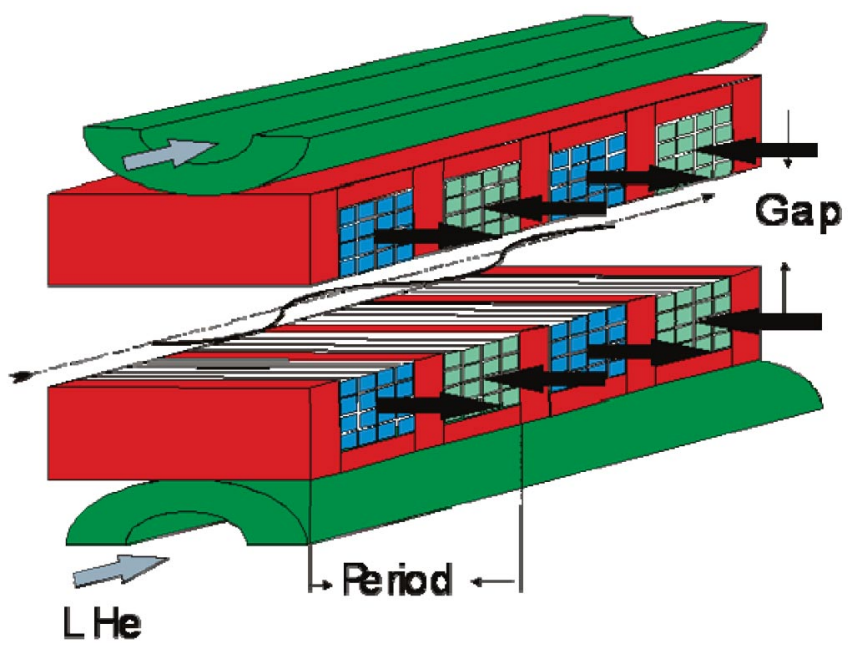

FIG. 1. (Color) Schematic layout of a superconductive invacuum undulator used for this experiment. The current direction in the wires alters. The undulator can be cooled indirectly either by liquid helium or a cryocooler. The arrows mark the direction of the current.
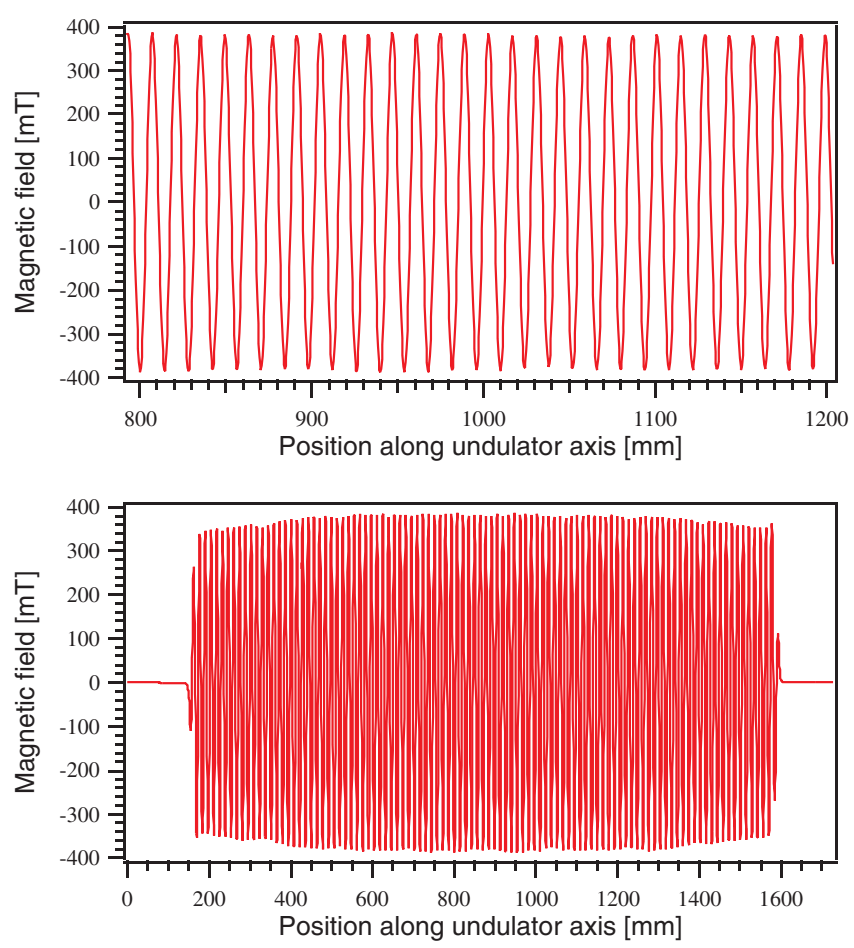

FIG. 2. (Color) (a) Example of the field measured (several periods are selected out of 100). Gap width $8 \mathrm{~mm}$, current density $500 \mathrm{~A} / \mathrm{mm}^{2}$. (b) The field of the full 100 periods. The coils were bent by $250 \mu \mathrm{m}$ when welded into the cryostat. In the future this welding process will be replaced by a different fixation in order to avoid this problem.

the extrema and $1^{\circ}$ in the average [12]. (Note that this definition of the phase error deviates from the conventional definition: usually the phase error describes the standard deviation of a statistical distribution [1] but this is a systematic error.) For future undulators the tool causing this "phase error" will be modified so that the phase error will hopefully be less than $1^{\circ}$.

Figure 2(b) shows the overall field distortion due to another mechanical error which happened during the integration of the undulator coils into the cryostat. As a result of tensions caused by the welding process both coils were bent at the ends. The deviation from flatness is about $250 \mu \mathrm{m}$. In the future the coils will not be welded anymore into the cryostat so this error will hopefully disappear. Nevertheless is was decided to use the coils as they were in order to obtain beam results as soon as possible.

The storage ring compatible cryostat is shown in Fig. 3. The undulator coils are cooled down by 3 Sumitomo cryocoolers to about $4 \mathrm{~K}$. A $300 \mu \mathrm{m}$ thick stainless foil with a $30 \mu \mathrm{m}$ copper coating is placed between the cold mass and the beam vacuum.

The $300 \mu \mathrm{m}$ foil separates the two independent vacuum systems: the so-called beam vacuum with only metallic and ceramic surfaces and the insulation vacuum system needed for the cryogenic operation. The position of the 


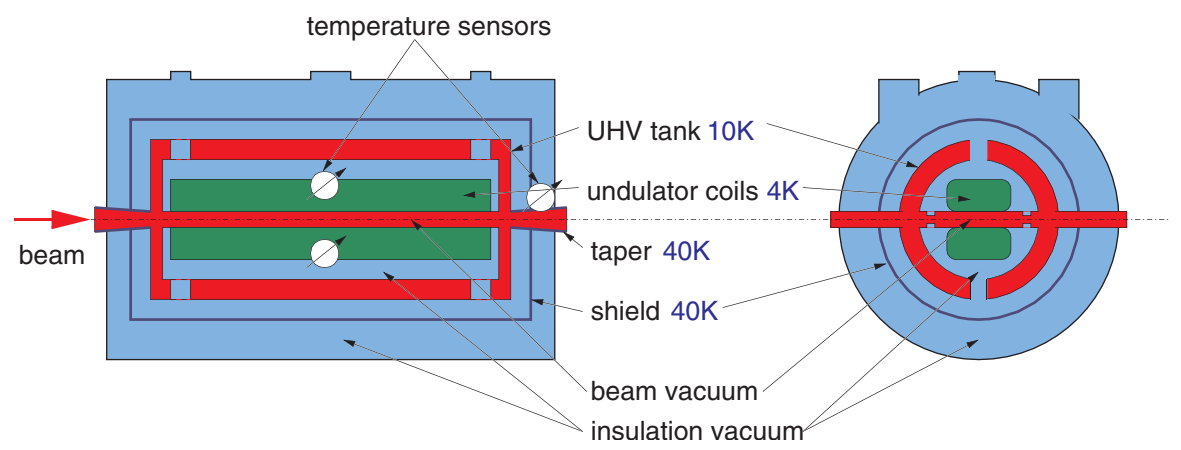

FIG. 3. (Color) Schematic layout of the superconductive undulator. The position of the temperature sensors are marked.

temperature sensors are marked in this schematic drawing: one at each coil (4 K level) and one at the taper (40 K level).

This device was the first one to be installed in a storage ring. Therefore a design was chosen which allows to operate the undulator with different magnetic gap widths: 16,12 , and $8 \mathrm{~mm}$ and open $(25 \mathrm{~mm})$. Recently for test purposes the vertical beta function in the undulator was reduced to $1 \mathrm{~m}$ which will allow to operate the undulator with a gap of $5 \mathrm{~mm}$ and a beam lifetime of more than $20 \mathrm{~h}$. In the near future this will be the mode of operation of the undulator.

The aim of the experiment was to determine the heat load by the beam between the two 4 to $5 \mathrm{~K}$ cold $\mathrm{Cu}$ surfaces. In principle the undulator can be heated by the beam via three mechanisms:

(i) heating by synchrotron radiation from upstream magnets,

(ii) heating by synchrotron radiation produced by the undulator,

(iii) heating by the high frequency image currents on the cold surface [2].

A collimator system is located in front of the undulator consisting of four independently movable collimators: two

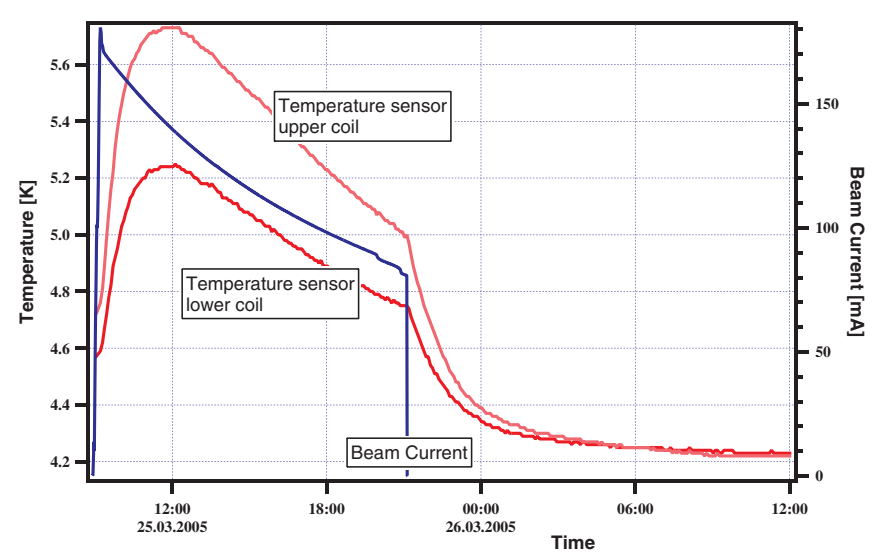

FIG. 4. (Color) Measured temperature change in the undulator device caused by the current (170 $\mathrm{mA}$ maximum beam current, beam energy $2.5 \mathrm{GeV}$ ). horizontal (inside and outside) and two vertical (upside and downside). The collimators are placed at a distance of approximately $1 \mathrm{~m}$ from the entry point of the undulator. Figure 4 shows the overall temperature rise caused by the beam. The gap width is $12 \mathrm{~mm}$ and the stored beam current is about $170 \mathrm{~mA}$ at the beginning (beam energy $2.5 \mathrm{GeV}$ ).

The beam position inside the undulator is not centered during this run. This can be seen by the fact that the temperature is different in the upper and the lower coil. The maximum temperature rise is about 0.6 and $1 \mathrm{~K}$. In order to establish the reasons for the rise in temperature the following experiments were performed.

The storage ring was filled with $40 \mathrm{~mA}$ at $2.5 \mathrm{GeV}$. After $1 \mathrm{~h}$ the coil temperature was stable at an equilibrium value of $4.8 \mathrm{~K}$ (Fig. 5). The undulator gap was $16 \mathrm{~mm}$. Afterwards the undulator current was switched on and the current through the undulator was changed in steps up to $600 \mathrm{~A}$. The generated synchrotron radiation causes only slight changes to the temperature in the coils. This experiment was repeated with a gap of $8 \mathrm{~mm}$. The results were similar.

The heating by synchrotron radiation produced by the undulator [item (ii) in the previously mentioned table] cannot explain the temperature change seen in Fig. 4. For

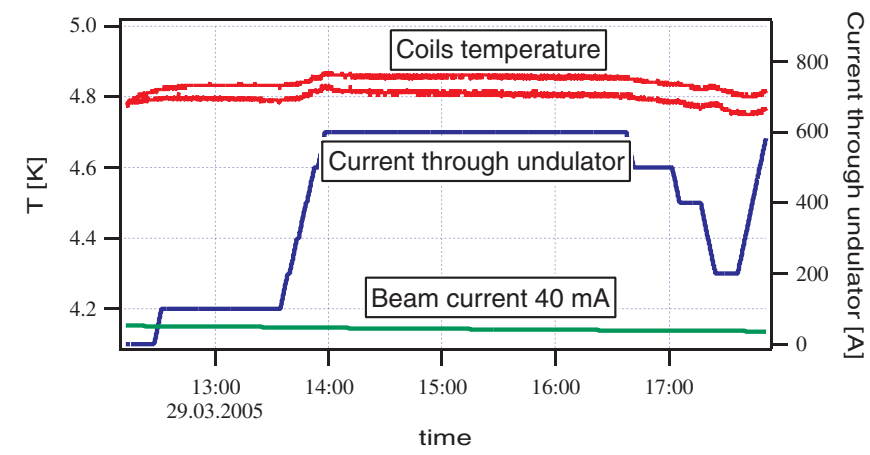

FIG. 5. (Color) Test to determine whether the synchrotron radiation produced by the undulator can heat cold surfaces. The gap is $16 \mathrm{~mm}$, the current through the undulator is $40 \mathrm{~mA}$ (lower curve). The undulator current is changed between 0 and $600 \mathrm{~A}$ in several steps. From this curve it becomes clear that the heating of the undulator by self produced synchrotron radiation is small. 
the same reasons effect (iii) (resistive wall heating) can be excluded. This is in good agreement with an earlier theoretical study [13]. The temperature rise is mainly caused by synchrotron radiation from the upstream bending magnet. Two experiments described in the following investigate these findings in more detail.

(A) Misalignment between the beam axis and the undulator axis can lead to a minor degree of heating as shown in Fig. 6. The measurements are performed with a $12 \mathrm{~mm}$ gap, the current is $40 \mathrm{~mA}$ and the energy $2.5 \mathrm{GeV}$. The first curve shows the temperature at the taper and the second one the temperature at the coils. Despite the fact that the current in the coils is increased, their temperature drops slowly during the run. At the end of the run the coils are forced into a quench. The quench does not pose any serious problem. Thanks to the bifilar character of the coil windings, the amount of stored energy is small. As a result of quenching the temperature rises to about $7 \mathrm{~K}$ and drops to its normal value (depending on the installed cooling capacity) within about half an hour. The first curve in Fig. 6 shows the temperature at the taper. Only a very small part of the synchrotron radiation generated hits the taper, thereby warming it by about $1.2 \mathrm{~K}$.

(B) If the heating is caused by synchrotron radiation from the upstream magnet, the effect must be strongly
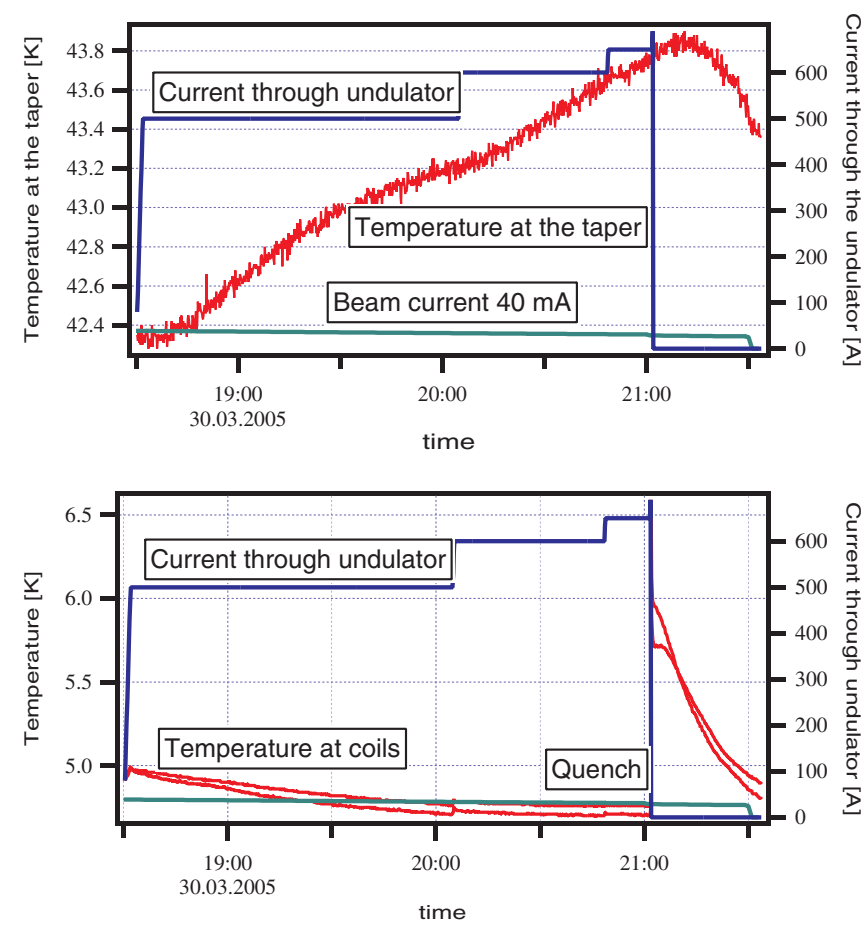

FIG. 6. (Color) The first graph shows that the $\mathrm{x}$-ray beam generated by the undulator hits the taper and warms it. In the second graph it is shown that this does not influence the temperature of the coils. At the end of the run the undulator is forced to quench. The recovery time is quite short and after about half an hour there is a return to the previous temperatures.
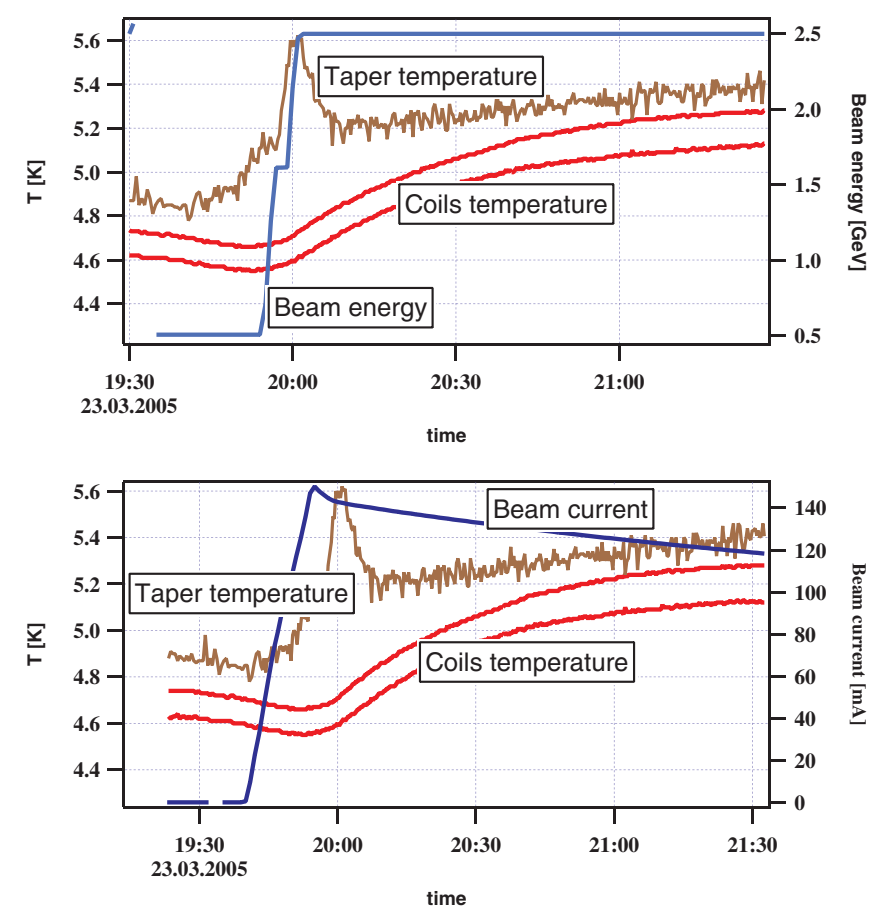

FIG. 7. (Color) The mechanism which heats the taper and the undulator coils seems to be different. The taper is heated both by the current (regardless of the energy) and the synchrotron radiation from the upstream bending magnets; the coils, on the other hand, are only affected by the synchrotron radiation from the upstream magnets and/or by thermal conductivity from the taper. Note that the taper temperature is about $40 \mathrm{~K}$ and this temperature is not indicated on the axis.

beam-energy dependent. This is shown in Fig. 7. At ANKA the beam is first stored at $0.5 \mathrm{GeV}$, then ramped to $1.6 \mathrm{GeV}$ and after a short interval to $2.5 \mathrm{GeV}$. This procedure is clearly evident from the first curve in Fig. 7. The taper temperature already begins to rise during filling despite the fact that it is exposed to very little synchrotron radiation from the nearby bending magnet due to the low energy. The coil temperature remains almost completely unaffected. At $1.6 \mathrm{GeV}$ the synchrotron radiation from the nearby bending magnet hits the taper, the temperature rises and the collimator is closed. Afterwards the temperature falls again. When the beam reaches $2.5 \mathrm{GeV}$ the coil temperature rises.

This behavior would seem to indicate that there are two processes involved in warming the undulator: the taper is heated by the current and the synchrotron radiation, the coils mainly by the synchrotron radiation from the nearby magnets and/or partly by thermal conductivity from the taper. A similar pattern was observed at ESRF in-vacuum undulators [14] kept at room temperature.

The heating by the synchrotron radiation generated by upstream magnets seems to be at the moment the most critical issue for the operation of the undulator. Higher 

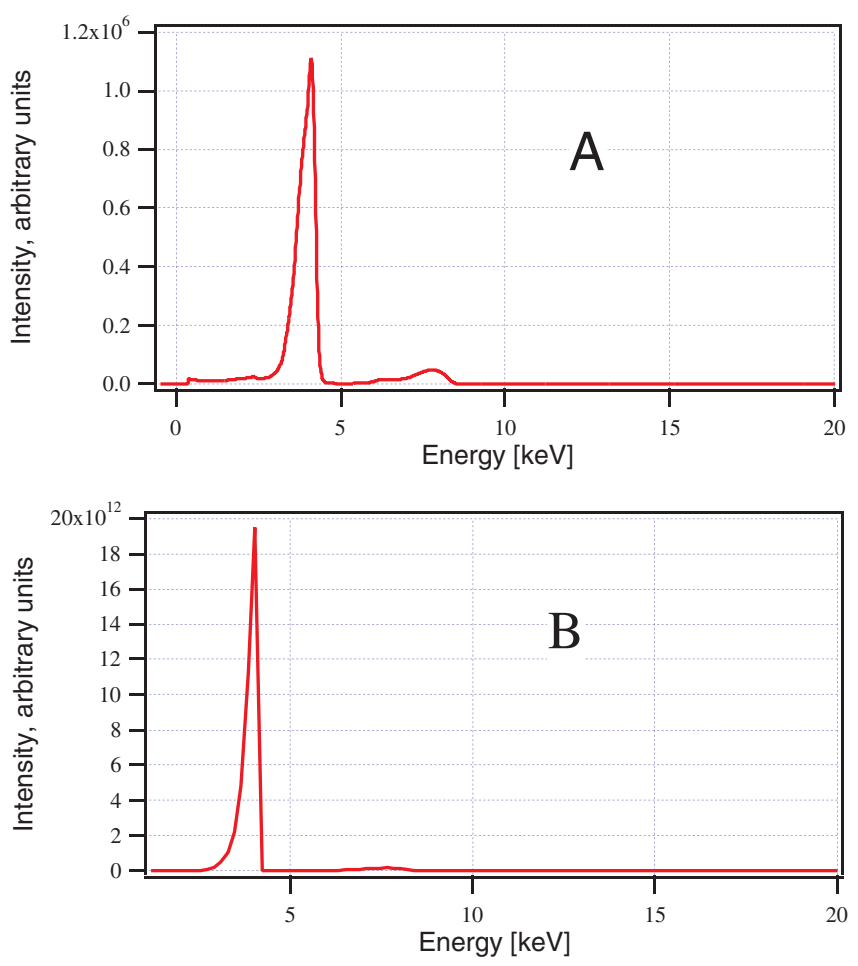

FIG. 8. (Color) Measured spectrum (a) of the emitted x rays and calculated spectrum with XURGENT [15] (assumed parameters: $\beta_{x}=25 \mathrm{~m}, \beta_{y}=9 \mathrm{~m}, \varepsilon_{x}=40 \mathrm{~nm}, \varepsilon_{y} / \varepsilon_{x}=10^{-2}$ ). The stored electron beam current was $112 \mathrm{~mA}$ and the $k$ value of the undulator was 0.17 .

temperatures at the superconducting wires mean a reduction of the maximum current and therefore a reduction of the maximum field. In a very rough estimate the synchrotron power from the upstream magnets is about $2.5 \mathrm{~W}$ at $100 \mathrm{~mA}$ (beam energy $2.5 \mathrm{GeV}$ ). This figure can be reduced in the following ways: first of all the undulator is at the moment only protected by a single upstream scraper which provides only limited protection for diffuse radiation, e.g., from quadrupoles. The single blade system therefore will be replaced by a collimator system in the near future. Second, the inner foil of the undulator has to be modified at future superconducting in-vacuum undulators so that the heat transport produced by the synchrotron radiation bypasses the wires.

Figure 8 shows a first measured spectrum of the $x$ rays (beam current $112 \mathrm{~mA}$ ). The x-ray beam is measured through a $50 \mu \mathrm{m}$ pinhole. The photons were measured via a scattering foil with a standard Si drift detector $8.3 \mathrm{~m}$ downstream from the undulator. This spectrum is in good agreement with calculations conducted with one of the well-known simulation programs, e.g., the program XURGENT [15].

In conclusion, it has been demonstrated that superconductive undulators are excellently suited for use in storage rings. The cold-bore temperature undergoes a slight in- crease mainly caused by the synchrotron radiation of the upstream bending magnet. The anomalous skin effect and the heating by the low energy part of the undulator radiation seems to be small. In addition it was found that a quench does not interfere with the stored beam. As a next step, a superconductive undulator similar to the one described in this paper will be installed at the ESRF within the framework of a European collaboration between ANKA, ELETTRA, ESRF, and MAXLAB.

\section{ACKNOWLEDGMENTS}

This work is partly supported by the BMBF Grant No. 806033/7 and the EU Grant No. RII3-CTT2004506008 IA-SFS within the 6th European framework program. The authors would like to thank Professor Maschuw and Professor Saile from Karlsruhe, Dr. Pascal Elleaume from the ESRF, Professor Moser from the National University of Singapore, and Professor Jochen Schneider from DESY for support and valuable discussions. The measurements at ANKA were performed together with the ANKA machine group: I. Birkel, E. Huttel, A.-S. Müller, and P. Wesolowski.

[1] Undulators, Wigglers and their Applications, edited by $\mathrm{H}$. Onuki and P. Elleaume (Taylor and Francis, London, 2003).

[2] G. E. Reuter and E. H. Sondheimer, Proc. R. Soc. A 195, 336 (1948). For an overview see also B. Podobedov, in Proceedings of the Workshop on Superconductive Undulators and Wigglers, Grenoble, France, 2003 (unpublished).

[3] James B. Murphy, BNL Report No. BNL 42333, 1996.

[4] T. Hara, T. Tanaka, H. Kitamura, T. Bizen, X. Marechal, and T, Seike, Phys. Rev. ST Accel. Beams 7, 050702 (2004).

[5] I. Ben-Zvi, Z. Y. Jiang, G. Ingold, and L. H. Wu, Nucl. Instrum. Methods Phys. Res., Sect. A 297, 301 (1990).

[6] H. O. Moser, B. Krevet, and H. Holzapfel, Research Center Karlsruhe, German Patent No. DE 4101094.

[7] A. Geisler, A. Hobl, D. Krischel, R. Rossmanith, and M. Schillo, IEEE Trans. Appl. Supercond. 13, 1217 (2003).

[8] S. Prestemon et al,, in Proceedings of the Particle Accelerator Conference, Portland, OR, 2003 (IEEE, Piscataway, NJ, 2003), p. 1032; Joint Accelerator Conferences Website, http;//accelconf.web.cern.ch/ accelconf/

[9] T. Hezel et al., in Proceedings of the Particle Accelerator Conference, New York, 1999 (IEEE, Piscataway, NJ, 1999), http://accelconf.web.cern.ch/accelconf/

[10] http://www.lightsources.org/cms/?pid=1000121

[11] A. Bernhard, B. Kostka, R. Rossmanith, T. Schneider, D. Dölling, A. Hobl, D. Krischel, S. Kubsky, U. Schindler, and E. Steffens, in Proceedings of the European Particle Accelerator Conference, Lucerne, 
2004 (EPS-AG, Lucerne, 2004), http://accelconf. web.cern.ch/accelconf

[12] D. Wollmann, Diploma thesis, University of Dresden, 2005 (unpublished).

[13] S. Strohmer, in Proceedings of the Workshop on Superconductive Undulators and Wigglers, Grenoble
(France), 2003 (unpublished), http://www.esrf.fr/ Accelerators/Conferences/ID_Workshop/

[14] P. Elleaume, ESRF (private communication).

[15] R. P. Walker and B. Diviacco, Rev. Sci. Instrum. 63, 392 (1992). 